\title{
NUCLEONICA: a nuclear science portal
}

\author{
J. Magill ${ }^{1, \text { a }}$, J. Galy ${ }^{1}$, R. Dreher ${ }^{1}$, D. Hamilton ${ }^{1}$, M. Tufan ${ }^{1}$, C. Normand ${ }^{1}$, A. Schwenk-Ferrero ${ }^{2}$, and H.W. Wiese ${ }^{2}$ \\ 1 European Commission, DG Joint Research Centre, Institute for Transuranium Elements, Postfach 2340, 76125 Karlsruhe, Germany \\ ${ }^{2}$ Forschungszentrum Karlsruhe Technik und Umwelt, Postfach 3640, 76021 Karlsruhe, Germany
}

\begin{abstract}
NUCLEONICA is a new nuclear science web portal from the European Commission's Joint Research Centre. The portal provides a customisable, integrated environment and collaboration platform for the nuclear sciences using the latest internet "Web 2.0" dynamic technology. NUCLEONICA is aimed at professionals, academics and students working with radionuclides in fields as diverse as the life sciences (e.g., biology, medicine, agriculture), the earth sciences (geology, meteorology, environmental science) and the more traditional disciplines such as nuclear power, health physics and radiation protection, nuclear and radio-chemistry, and astrophysics. It is also used as a knowledge management tool to preserve nuclear knowledge built up over many decades by creating modern webbased versions of so-called legacy computer codes.
\end{abstract}

\section{Introduction}

Due to a general lack of interest in nuclear power over many years, particularly in Europe, there has been a gradual decline in the nuclear skills base. The nuclear field has become very much a "grey-haired profession" with an ageing population of nuclear professionals. Today, as a result of recent developments on issues such as energy security and protection of the environmental, we are witnessing a resurgence of interest in nuclear power. In order to support this development we will need a nuclear skills renaissance in education, training, and knowledge management in the nuclear field.

There is also large range of "non-power" applications of radioisotopes and radiation in a variety of diverse fields such as medicine (e.g., cancer therapy), agriculture (e.g., pest population control), food irradiation (e.g., to increase shelf-life) and in industry (e.g., tracers, radiography, gauging, radiation processing, etc.), where such nuclear skills are also required.

To support this renaissance, we have developed a nuclear skills "toolbox" using the latest internet technology. This toolbox can be used for daily activities by experts, teachers, postgraduates and students of nuclear science. The nuclear skills toolbox proposed above takes the form of a Nuclear Science Web Applications Portal: NUCLEONICA [1] to encompass the knowledge of generations of nuclear scientists (in the form of databases, software applications, etc.) and make this available in a modern, user-friendly way which is fast, accurate and cost effective using the latest internet technology. In particular NUCLEONICA consists of an integrated environment and collaboration platform through the internet (databases, software applications, training courses) providing a one-stop, user friendly, fast and accurate nuclear science information source to allowe experts and non-experts alike to concentrate on scientific results rather than tedious background chores.

\footnotetext{
${ }^{a}$ Presenting author, e-mail: Joseph. Magill@ec. europa.eu
}

\section{NUCLEONICA innovative features}

NUCLEONICA makes use of cutting-edge internet technologies such as AJAX (Asynchronous JavaScript and XML) and offers security enhanced "software as a service" (SaaS) on the web rather than software on a PC thus avoiding problems of software licensing, installation, updates, etc. The web applications are browser and operating system independent and can be accessed with Internet Explorer, Mozilla-based browsers (Mozilla, Firefox, Netscape) and a variety of other browsers such as Opera, Safari, etc. Another Web 2.0 feature is the Personalised Desktop with detailed user rights configurable via administration menu. A Mobile Device Portal is also available for a variety of mobile devices.

NUCLEONICA applications are designed to be very user friendly, intuitive, and require a minimum of learning time. These powerful applications, which form the "backbone" of the nuclear science toolbox, can be used by professionals and students for everyday calculations. For advanced users, who prefer a more "hands on" approach, NUCLEONICA provides this with its advanced scripting interface which gives the user a powerful programming interface. The data used in the calculations are taken from international datafiles (see fig. 2) and presented in the form of user-friendly databases with fast and powerful search functions.

Computer codes which have taken many man-years of development (e.g., legacy software) and have been written in a variety of programming languages will run on the NUCLEONICA web-server (as dynamic linked libraries, DLLs). The user can input data to these applications with the use of a web browser and the use of active server pages (ASPs). The information (output) is returned to the user also in the form of ASPs. The user does not "see" or need to worry about the language in which the application has been written. This allows an extremely user-friendly approach to obtaining information and solutions with almost no learning time for the underlying application. An example of such a development is webKORIGEN for nuclear fuel depletion and decay calculations based on KORIGEN [2]. The KORIGEN code, developed in the Karlsruhe Research Centre, is a stand alone package which 


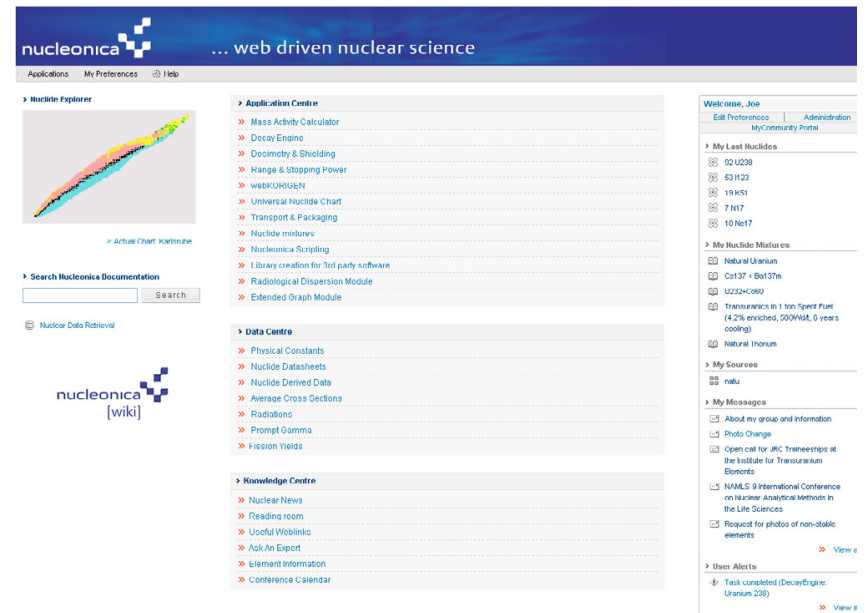

Fig. 1. The NUCLEONICA web portal showing access to the Application, Data, and Knowledge Centres. NUCLEONICA will keep track of user activities by customising (remembering and storing) these in a Personalized Desktop.

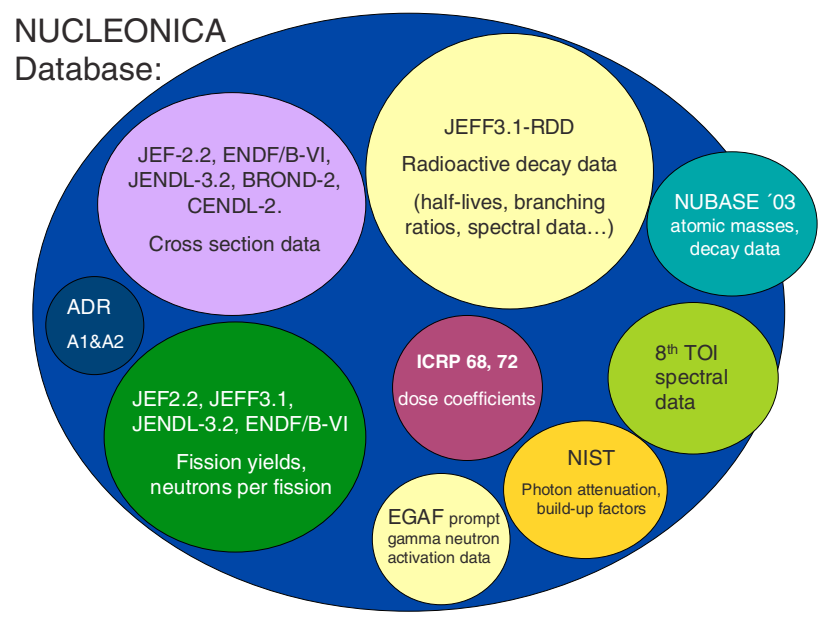

Fig. 2. Structure of the NUCLEONICA database.

serves to calculate the fuel depletion, burn-up and decay. KORIGEN, originating from the ORNL ORIGEN code, is used in the German nuclear industry and by German licensing authorities to determine the integral characteristics of spent nuclear fuel as nuclide compositions, radioactivities, decay heat, radiation sources and radiotoxicities. The KORIGEN supervising staff at FzK together with the NUCLEONICA developers at ITU created a web-based version of KORIGEN called webKORIGEN - for use in NUCLEONICA. In order to facilitate the input preparation, preprocessing, running, postprocessing and fast graphical output generation for a set of standardized KORIGEN solved problems, webKORIGEN is trimmed to three major classes of nuclear plants: Pressurized Water Reactors (PWR), Boiling Water Reactors (BWR), and the European Fast Reactor (EFR) (a future extension to current industrial technology).

Extensive use is made of "wiki" technology [3] in the NUCLEONICA knowledge centre for online Help, Articles, Weblinks, etc. This is discussed in more detail in section 5 .

News aggregation services, in which web-crawlers are used to "crawl" newspapers and deliver news to a web browser, are attracting considerable attention. These are computer generated news pages based on the use of web crawler technology. Web crawlers are used to search thousands of news websites to look for information on a particular subject. NUCLEONICA's web crawlers search the internet for nuclear science news. This may be of a political nature through to finding the latest conferences on nuclear science. These services are based on XML and RSS feeds using JRC webcrawler technology.

\section{NUCLEONICA Portal}

NUCLEONICA offers the following main features:

Application Centre: Application modules (decay engine, dosimetry and shielding, fission yields, range \& stopping power, reactor irradiation, transport and packaging, etc.) with publication quality graphics. An advanced scripting language is available for user defined calculations and batch processing.

Data Centre: Online interactive nuclide charts, reference data and searchable databases for internationally evaluated nuclear data and library creation software.

Knowledge Centre: Extensive use is made of "wiki" technology in the NUCLEONICA knowledge centre for online Help, Articles, Weblinks, Discussion groups, etc. The ease of operation makes the NucleonicaWiki an effective tool for changing or updating content - this allows the portal to grow organically and provide a powerful nuclear science collaboration platform for its users. The nuclear news aggregation services, based on XML and RSS feeds using JRC web-crawler technology, provide latest news and information on nuclear issues.

Personalised Desktop: NUCLEONICA will keep track of your recent activities by "remembering" and storing these in a Personalized Desktop.

The key advantages for the users of NUCLEONICA can be summarised as follows:

- Manage all your data in a single browser-based system: The web applications are browser and operating system independent and can be accessed with Internet Explorer, Mozilla-based browsers (Mozilla, Firefox, Netscape) and a variety of other browsers such as Opera, Safari, etc.

- Don't waste time writing and testing programs: NUCLEONICA provides you with user friendly, reliable, and fast modules (for decay, dosimetry \& shielding, range and stopping power, transport and packaging, reactor irradiation calculations, etc.)

- Improve the quality of your work: avoid the tedious task of searching for nuclear data. NUCLEONICA uses the most recently evaluated nuclear data from international datafiles (JEFF3.1, $8^{\text {th }}$ Table of the Isotopes, ICRP72, etc.).

- Publication quality scientific graphs: at any time from any location. NUCLEONICA web driven graphics package is easy to use and delivers publication quality graphs in a variety of formats (GIF, JPG, PNG, EPS, EMF, PDF, SVG).

- Keep informed on nuclear developments: NUCLEONICA web crawlers scan hundreds of websites every few minutes to bring you the latest nuclear news. 
- Keep track of your recent activities: NUCLEONICA will "remember" your recent activities and store these in a Personalized Desktop with detailed user preferences.

- Provides the opportunity to introduce and share your expertise through our NucleonicaWiki - a collaborative authoring tool in nuclear science.

- Assistance in preparing a lecture or a training course: NUCLEONICA is an ideal source of information, articles, weblinks, graphics, tables, etc.

\section{NUCLEONICA database}

The NUCLEONICA database is based on the Joint Evaluated Fission and Fusion (JEFF3.1) radioactive decay datafile [4] which contains decay data on 3852 nuclides in ground and isomeric states. The relational nature of the NUCLEONICA database allows for fast searching and data retrieval in comparison to the non-relational JEFF3.1 Datafile. Moreover the NUCLEONICA database contains supplementary information on approximately 93 additional nuclides and their half-lives which are not listed in JEFF3.1 but are present in NUBASE '03 [5], extending the total number of nuclides (ground and isomeric states) to 3947. In addition, NUBASE '03 data on the atomic weights, binding energies, mass excesses, and abundances are included in the Nucleonica database (in the "materials" table).

To support the nuclear science applications in Nucleonica, the database is further complemented by a variety of data supplied by various sources:

- spectral data (energies, emission probabilities, etc.) from JEFF3.1. In addition, spectral data from the $8^{\text {th }}$ table of isotopes [6] can be selected for comparison and for library creation for gamma spectroscopy.

- photon mass attenuation coefficients and the mass energyabsorption coefficients from the National Institute of Standards and Technology [7].

- build-up factors, to model the scattering effects in the shield material for the dosimetry module which have been taken from [8].

- prompt gamma ray activation data [9] for thermal neutron activation analysis.

- fission yield data from the main international datafiles: JEF2.2, JEFF3.1 [4], JENDL-3.2 [10], and ENDF/BVI $[11,12]$.

- integral cross section data from JEF Report 14 [13] which contains averaged neutron cross-sections from international datafiles JEF-2.2, ENDF/B-VI, JENDL-3.2, BROND-2, and CENDL-2.

- effective dose coefficients for ingestion and inhalation, $e(50)$, have been taken from the International Commission for Radiological Protection compilation [14].

- the A1 and A2 activity and activity exemption limits for packaging and transportation [15].

- properties of the elements [16] (densities, melting points, boiling points, etc.).

- tables of physical constants and conversation factors [17].

- miscellaneous tables to manage user data.

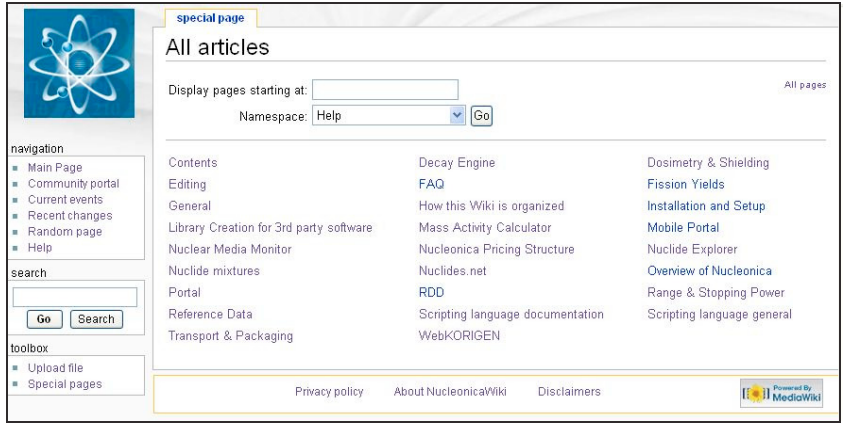

Fig. 3. The NucleonicaWiki: Wiki-based online user manual.

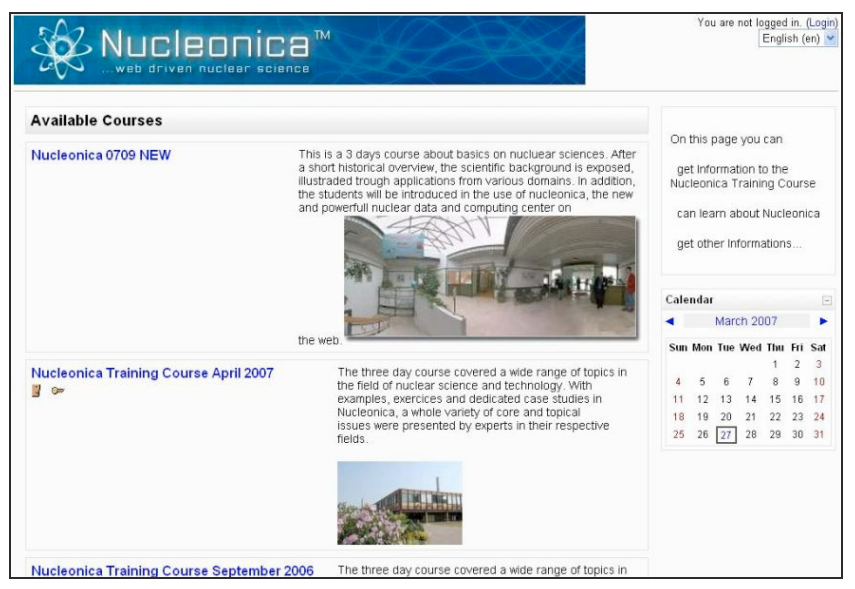

Fig. 4. The NUCLEONICA e-learning platform blends internet technology with traditional teaching methods.

\section{NUCLEONICA Wiki}

The NucleonicaWiki [2] allows the development team to add, remove, edit and change content on the portal using wiki technology - an approach which is extremely flexible. The NUCLEONICA administrators can determine which parts of the portal can be updated and by whom. Certain parts of the portal will only be accessible to the administrators. Wikis are not only restricted to the online manual but can be used for adding articles and links, discussions groups, etc. This will allow NUCLEONICA to grow organically and provide a powerful nuclear science collaboration platform for its users.

\section{NUCLEONICA training courses \& e-learning}

The web supported training courses will be based on the use of "Moodle" - an open source e-learning platform specially designed to create online courses with opportunities for rich interaction.

The courses introduce the basic concepts of nuclear science and technology and are suitable for participants from the nuclear industry, nuclear research organizations, universities, regulatory authorities and nuclear medicine institutes. Core topics range from the history of radioactivity, nuclide charts, the Karlsruhe nuclide chart [18], radiation protection and health physics through to the storage and transportation of radioactive materials. Recent exciting developments in nuclear 
science are highlighted in a series of special topics covering nuclear forensic science/illicit trafficking, nuclear science with high intensity lasers, environmental radioactivity, radiation hormesis and the LNT hypothesis, etc. Lectures are be followed by a series of "hands-on" case studies based on the use of the Nuclides.net/NUCLEONICA web-based applications to give the user direct experience in the above areas.

\section{References}

1. The NUCLEONICA web site: http://www.nucleonica.net

2. KORIGEN see: U. Fischer, H. Wiese, ORNL-tr-5043, 1983 also KfK 3014. For webKORIGEN in NUCLEONICA, see: http://www.nucleonica.net:81/wiki/index.php/Help:WebKORIGEN

3. NucleonicaWiki: http://www.nucleonica.net:81/wiki/index.php/ Main_Page

4. A. Koning et al., JEFF Report 21: The JEFF-3.1 Nuclear Data Library (OECD, 2006).

5. G. Audi et al., Nucl. Phys. A 729, 3 (2003) (http://amdc.in2p3.fr/web/nubase_en.html).

6. R.B. Firestone, Table of Isotopes, 8th edn. (John Wiley \& Sons Inc., New York, updated in 1998).

7. J.H. Hubbel, S.M. Seltzer, Radiat. Res. 136, 147 (1993), website at: http://physics.nist.gov/PhysRefData/ XrayMassCoef/cover.html
8. American National Standard for gamma-ray Attenuation Coefficients and Buildup Factors for Engineering Materials, ANSI/ANS-6.4.3-1991 (1991).

9. R.B. Firestone et al., The Evaluated Gamma-ray Activation File (EGAF), CP769, International Conference on Nuclear Data for Science and Technology, American Institute of Physics, AIP Conference Proceedings, 769 (2005).

10. T. Nakagawa et al., J. Nucl. Sci. Technol. 32, 1259 (1995).

11. T.R. England, B.F. Rider, Evaluation and Compilation of Fission Product Yields, LA-UR-94-3106 ENDF349 (1993).

12. P.F. Rose, ENDF/B-VI summary documentation, Report BNLNCS-17541 (ENDF-201) (1991).

13. JEF Report 14: Table of Simple Integral Neutron Cross-Section Data from JEF-2.2, ENDF-BVI, JENDL-3.2, BROND-2 and CENDL-2 (OECD, 1994).

14. Age-dependent Doses to Members of the Public from Intake of Radionuclides: Part 5, Compilation of Ingestion and Inhalation Dose Coefficients, ICRP PUBLICATION 72, Annals of the ICRP 26 (Pergamon Press, 1996).

15. ADR (from Accord Dangereuses Route) http://en.wikipedia.org/wiki/European_Agreement_concerning the_International_Carriage_of_Dangerous_Goods_by_Road

16. Handbook of Chemistry and Physics on CD-ROM, version 2006, edited by D.R. Lide (CRC Press, Boca Raton, Florida, Sept. 2005).

17. P.J. Mohr, B.N. Taylor, Rev. Mod. Phys. 77, 1 (2005).

18. J. Magill, G. Pfennig, J. Galy, Karlsruher Nuklidkarte, 7th edn. (2006), see www.nucleonica.net. 